\title{
DOENÇA DE CROHN GASTRODUODENAL - relato de quatro casos e revisão da literatura
}

\author{
Luiz João ABRAHÃ O Jr", Luiz João ABRAHÃ ${ }^{* *}$, \\ Cleber VARGAS $^{* * *}$, Vera CHAGAS ${ }^{* * * *}$ e Homero FOGAÇA ${ }^{* * *}$
}

RESUMO - Racional - A doença de Crohn pode acometer todo o tubo digestivo, porém o envolvimento gastroduodenal é classicamente descrito como raro (0,5\% a 13\%). Objetivos - Descrever os achados clínicos, radiológicos, endoscópicos e o tratamento de quatro pacientes com doença de de Crohn gastroduodenal e rever a literatura. Pacientes e Métodos Quatro pacientes (um homem de 24 anos e três mulheres de 37, 66 e 74 anos) com epigastralgia, emagrecimento e febre baixa foram acompanhados nos hospitais das Universidades Federais do Rio de Janeiro e Fluminense. Diarréia intermitente e artralgia/artrite estavam presentes em dois e uma paciente foi operada de urgência por síndrome de obstrução pilórica. Exames laboratoriais revelaram anemia em uma das pacientes, sendo normais nos demais. Os estudos baritados revelaram ulcerações aftóides no estômago e duodeno, assim como ulcerações em íleo terminal e deformidades em ambos. Os achados endoscópicos compreenderam úlceras aftóides e serpiginosas e lesões polipóides no antro gástrico e úlceras geográficas em duodeno. Colonoscopia foi realizada em dois pacientes, revelando ileite ulcerada em um e pancolite ulcerada em outro. O exame histopatológico revelou processo inflamatório crônico inespecífico sem granulomas, sendo excluidas outras causas de doença granulomatosa gástrica. Instituído tratamento com prednisona e inibidores de bomba de prótons, com remissão da doença em dois pacientes e evolução para síndrome de estenose pilórica em um (submetido a gastroenteroanastomose). Conclusão - A doença de Crohn gastroduodenal possui características clínicas, terapêuticas e prognósticas distintas. O avanço da endoscopia digestiva e a adoção de novos critérios para o diagnóstico histopatológico tem demonstrado incidência maior (17 a 75\%) que a previamente relatada.

DESCRITORES - Doença de Crohn. Gastropatias. Duodenopatias.

\section{INTRODUÇÃO}

A doença de Crohn (DC) é doença inflamatória intestinal crônica transmural, de etiologia desconhecida, que pode acometer qualquer parte do tubo digestivo, da boca ao ânus, embora freqüentemente se localize no segmento ileocólico ${ }^{(2,9,10,34)}$.
Caracteriza-se pelo envolvimento segmentar e assimétrico (lesões salteadas), com tendência à recurrência, mesmo após ressecções cirúrgicas.

Não raramente se acompanha de manifestações extraintestinais, tais como artrite, uveíte, episclerite, eritema nodoso, pioderma gangrenoso, colangite esclerosante e espondilite

\footnotetext{
Serviços de Gastroenterologia e Anatomia Patológica do Hospital Universitário Clementino Fraga Filho - Universidade Federal do Rio de Janeiro - UFRJ, Rio de Janeiro, RJ., e Serviço de Gastroenterologia do Hospital Universitário Antônio Pedro - Universidade Federal Fluminense - UFF, Niterói, RJ.

* $\quad$ Mestrando em Gastroenterologia da UFRJ.

** Professor Titular de Gastroenterologia da UFF.

*** Professor Adjunto de Gastroenterologia da UFRJ.

**** Professor Adjunto de Anatomia Patológica da UFRJ

Endereço para correspondência: Dr. Luiz João Abrahão Junior - Praia de Icaraí, 299/502, Icaraí - 24230-004 - Niterói, RJ. e-mail: ljabrahao@brfree.com.br
} 
anquilosante, havendo inclusive relatos de acometimento da vesícula biliar $^{(30)}$ e do pâncreas ${ }^{(13)}$.

O envolvimento gastroduodenal foi inicialmente relatado por Gottlieb em 1937, sendo classicamente descrito como raro, com incidência de 0,5 a $13 \%$ em estudos retrospectivos de pacientes sintomáticos com doença ileocolônica submetidos a endoscopia digestiva alta ou seriografia ${ }^{(6,14,24,31,37)}$.

Mais recentemente, estudos prospectivos incluindo pacientes sintomáticos e assintomáticos, têm reportado incidência de $17 \%$ a $75 \%$ de envolvimento gastroduodenal ${ }^{(1,19,33)}$.

A raridade do envolvimento gastroduodenal sintomático pela DC, a necessidade de maior compreensão da doença, assim como suas características clínicas, terapêuticas e prognósticas distintas motivaram este relato.

\section{PACIENTES E MÉTODOS}

Foram acompanhados quatro pacientes com DC gastroduodenal: três provenientes do Hospital Universitário Clementino Fraga Filho, Universidade Federal do Rio de Janeiro e um do Hospital Universitário Antônio Pedro, Universidade Federal Fluminense, no ano de 1998, três do sexo feminino e um do masculino, com média de idade de 68 $\operatorname{anos}(27$ a 74 anos).

O tempo médio para o diagnóstico da doença foi de 11,3 meses ( 4 a 24 meses) e os sintomas de apresentação mais freqüentes foram dor epigástrica, vômitos, emagrecimento, presente nos quatro, e diarréia em dois. Um paciente apresentava sintomas sistêmicos como febre e poliartralgias e em dois houve rápida evolução para síndrome de estenose pilórica, refletindo complicação associada.

Em dois pacientes o diagnóstico inicial foi de doença péptica e nos dois restantes pancreatite aguda.

Todos foram submetidos a endoscopia digestiva alta, havendo predomínio do envolvimento da segunda porção duodenal que exibia lesões nos quatro pacientes. Nos pacientes com lesões gástricas associadas $(n=4)$, houve predomínio antral, estando a mucosa do corpo e fundo livre de lesões em todos os pacientes. Em apenas um foi encontrada alteração esofagiana (erosões longitudinais no terço distal), atribuída à doença do refluxo gastroesofágico.

As lesões gástricas mais prevalentes foram enantema isolado em dois, erosões planas em um, irregularidade mucosa em um, úlceras serpiginosas e nodulações da mucosa em um (Figura 1). No duodeno, os achados compreenderam úlceras bulbares se estendendo até a segunda porção (Figura 2), com estenose da luz em três (Figura 3) e deformidade bulbar isolada em um. As biopsias foram obtidas das áreas de mucosa lesada, não sendo retirados fragmentos de mucosa macroscopicamente normal.

Dois pacientes foram submetidos a colonoscopia que revelou pancolite ulcerada em um (com íleo terminal normal) e ileíte com envolvimento da válvula ileocecal (estando o cólon normal) em outro.

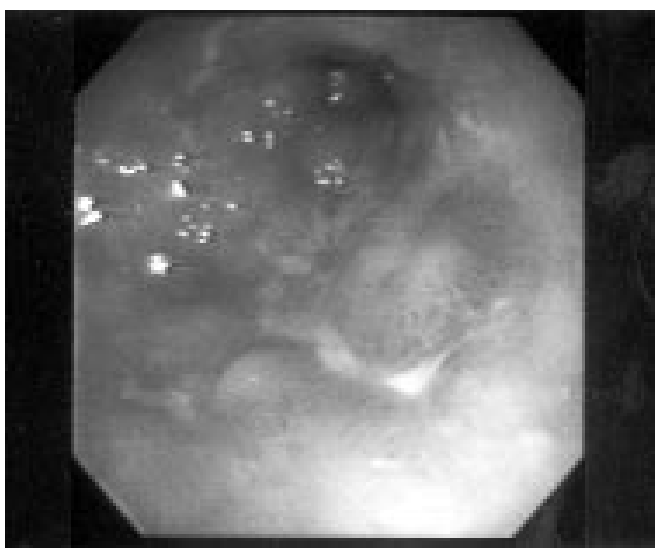

FIGURA 1 - Úlceras serpiginosas em antro, ocupando todas as paredes e associadas à nodulações

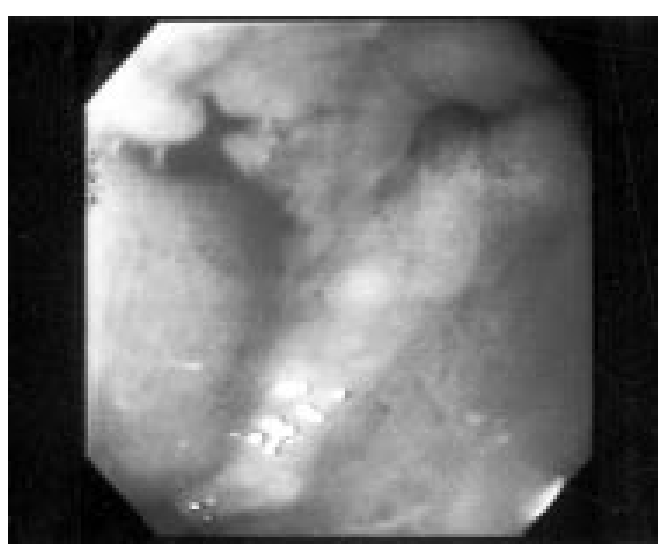

FIGURA 2 - Bulbo intensamente deformado com grande úlcera geográfica recoberta por fibrina que se estende à segunda porção

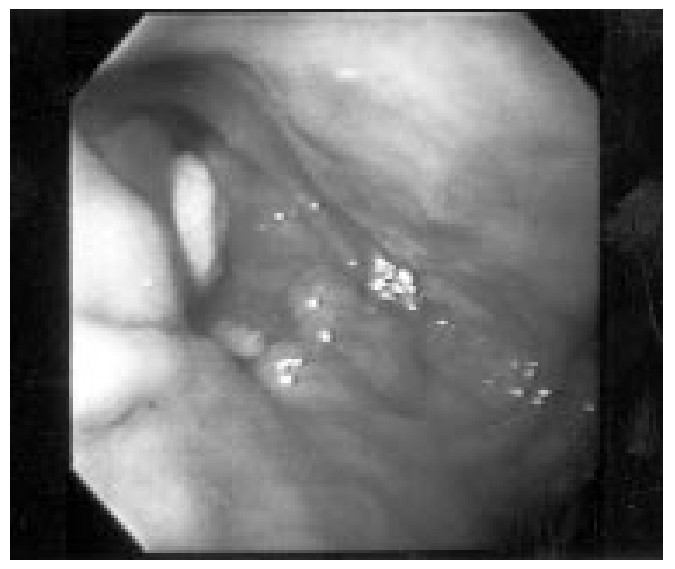

FIGURA 3 - Segunda porção duodenal, logo abaixo da papila de Vater, com estenose cerrada da luz e ulceração pouco profunda, de bordos elevados e nodulares, vista apenas em sua porção proximal 
A seriografia esôfago-estômago-duodeno foi realizada em três pacientes e os achados foram: aumento do volume gástrico em dois (Figura 4), espessamento do pregueado mucoso gastroduodenal em dois, úlceras aftóides de antro em um (Figura 5), úlcera em segunda porção duodenal em um (Figura 6), estenose de antro em um, divertículo em terceira porção duodenal com estenose de segunda e terceira porções duodenais em um. Três pacientes realizaram trânsito de delgado, que foi normal em dois e exibia estenose do íleo terminal com dilatação à montante e formação de pseudodivertículo em um.

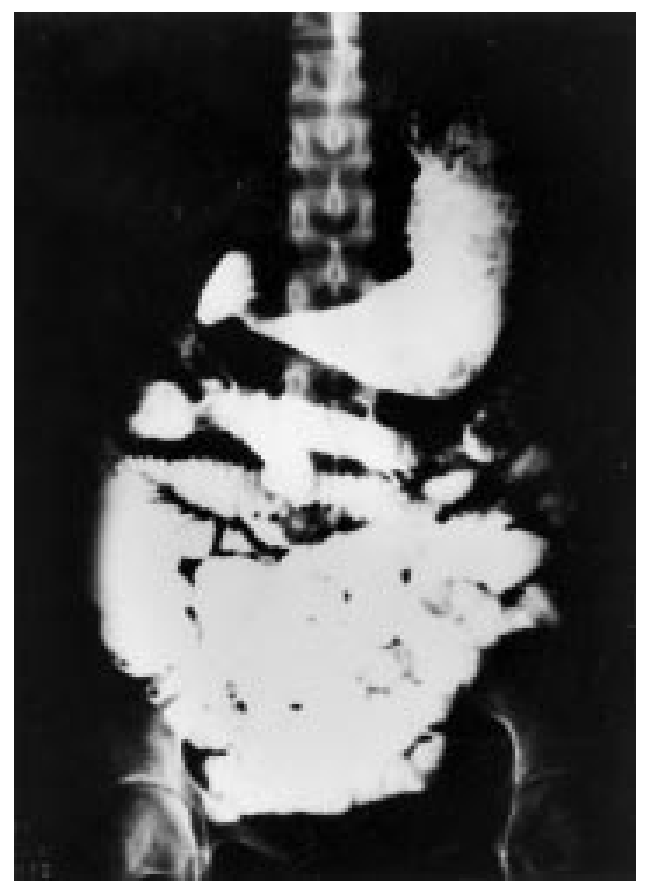

FIGURA 4 - Estômago de volume aumentado, com resíduos alimentares, distensibilidade do antro reduzida e irregularidade da mucosa

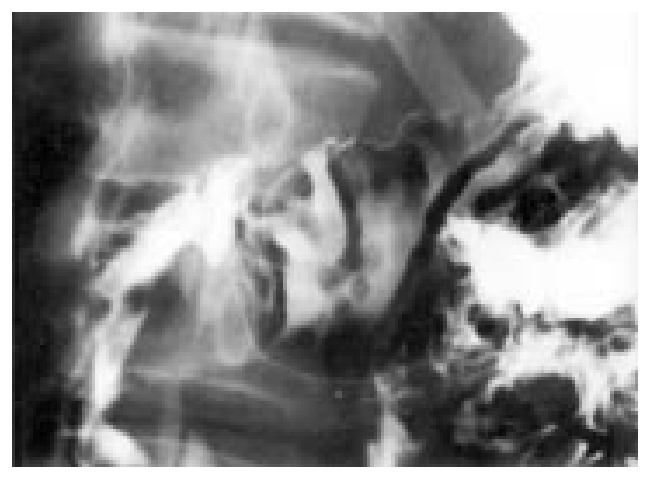

FIGURA 5 - Espessamento do pregueado mucoso antral com úlceras aftóides

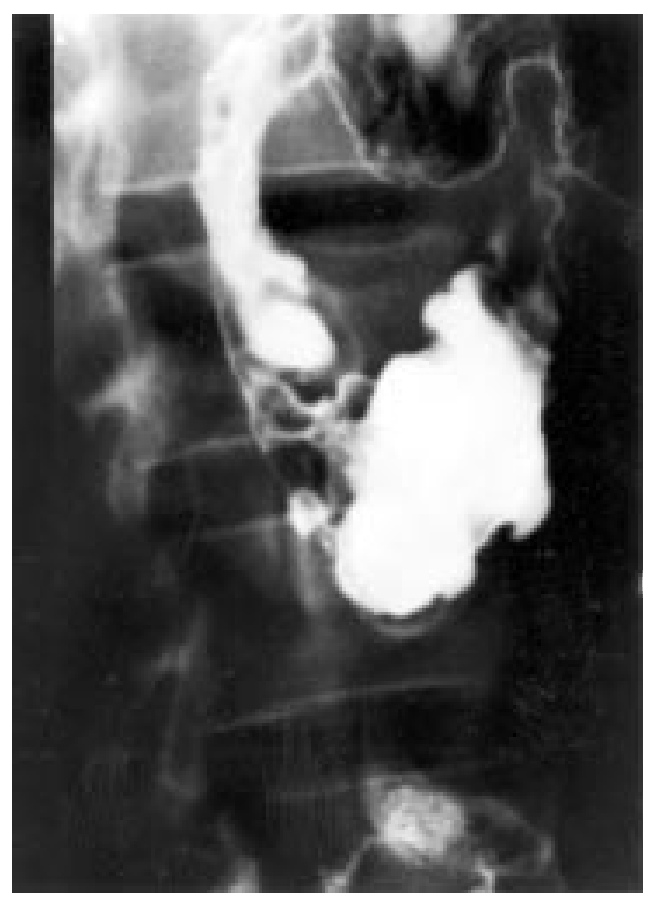

FIGURA 6 - Grande úlcera na segunda porção do duodeno

O exame histopatológico das biopsias gástricas revelou infiltrado inflamatório ativo em todos os casos, com ulceração em um; nenhum granuloma foi encontrado. Em um paciente, a biopsia obtida da segunda porção duodenal foi compatível com metaplasia gástrica total. Nenhum paciente apresentava infecção pelo Helicobacter pylori (um deles havia tratado 1 ano antes).

Todos os pacientes foram inicialmente tratados com corticoterapia (equivalente a $1 \mathrm{mg} / \mathrm{kg}$ de prednisona) e drogas antisecretoras (bloqueadores $\mathrm{H}_{2}$ ou inibidores da bomba de prótons) com bons resultados em três. Em um caso houve rápida evolução para síndrome de estenose pilórica, sendo submetido a cirurgia de urgência (gastroenteroanastomose). Dos três pacientes controlados clinicamente, dois permanecem em remissão corticóide-dependente e um desenvolveu síndrome de estenose pilórica necessitando ser operado.

\section{DISCUSSÃO}

Os quatro casos descritos representam uma forma rara de apresentação da DC, com envolvimento gastroduodenal predominante e que representa apenas $3 \%$ do total de pacientes com DC acompanhados no Hospital Universitário Clementino Fraga Filho, Universidade Federal do Rio de Janeiro desde a sua inauguração em 1978 (130 pacientes) $)^{(29)}$.

Sua real prevalência permanece desconhecida, sendo classicamente descrita de $0,5 \%$ a $13 \%$ dos pacientes ${ }^{(9,10,24,21)}$; porém, o avanço dos 
métodos radiológicos e principalmente dos endoscópicos, ocorrido nos últimos anos, permitiu o diagnóstico de formas precoces de DC gastroduodenal, atingindo prevalência de $20 \%$ a $76 \%$ em alguns estudos $^{(26,35)}$.

Clinicamente se caracteriza por dor epigástrica, náuseas, vômitos e emagrecimento, acometendo pacientes mais jovens que a DC ileocólica e ocorrendo, geralmente, em pacientes com DC distal previamente estabelecida, podendo precedê-la ou se manifestar isoladamente. Quando comparados aos pacientes com DC distal, apresentam o mesmo número de intervenções cirúrgicas, porém, com ressecções mais extensas ${ }^{(38)}$.

Devido a grande diversidade nos critérios diagnósticos da DC proximal, NUGENT e ROY ${ }^{(20)}$ propuseram, em 1989, os seguintes critérios, adotados desde então: 1- presença histológica de granulomas não-caseosos no trato gastrointestinal superior (TGS), sem evidências de doença sistêmica granulomatosa e/ou; 2- DC de intestino delgado ou grosso e achados radiológicos e/ou endoscópicos de inflamação difusa do TGS consistente com DC.

Os achados radiológicos na DC proximal se assemelham aos do envolvimento ileocólico e se dividem em estenóticos e não-estenóticos. $\mathrm{Na}$ fase não-estenótica pode-se observar espículas na mucosa (úlceras rasas puntiformes), hipertrofia de pregas, úlceras aftóides, pseudodivertículos, nodularidade, úlceras profundas ou aspecto em paralelepípedo $^{(21,35)}$. Na fase estenótica pode-se encontrar sinais característicos da doença tais como sinal do chifre de carneiro (obstrução do canal pilórico com rigidez e estenose do antro), sinal do pseudoBilroth I (estenose abrupta do antro sem identificação do piloro), estenose isolada da segunda porção duodenal e doença de duodeno distal contígua à doença jejunal ${ }^{(23)}$. A formação de fístulas, embora rara, pode ser apreciada no exame radiológico ${ }^{(31)}$.

O exame endoscópico pode revelar alterações tais como erosões, úlceras lineares ou aftóides, nódulos, espessamento de pregas, eritema em placas ou estenoses nos casos mais graves ${ }^{(1,7,24)}$, sendo o duodeno o segmento mais freqüentemente acometido, seguido pelo antro gástrico. Pouca atenção tem sido dada aos achados endoscópicos do corpo gástrico devido à raridade do seu envolvimento, sendo descrito recentemente o aspecto de "nó de bambu" por YOKOTA et al. ${ }^{(41)}$, e que consiste em pregueado de pequena curvatura de corpo espessado, cortado transversalmente por sulcos ou erosões. A presença destas lesões correlacionou-se com granulomas nas biopsias em $64 \%$ dos pacientes com erosões e $20 \%$ daqueles com apenas sulcos.

O exame histopatológico da mucosa gastroduodenal com lesões endoscópicas quase sempre revelará infiltrado inflamatório crônico, porém, somente o achado de granuloma epitelióide não-caseoso permitirá a confirmação do diagnóstico de DC. Estes são encontrados em $7 \%$ a $22 \%$ dos $\operatorname{casos}^{(7,19)}$, havendo relatos mais recentes de $68 \%{ }^{(31)}$ a $83 \%{ }^{(36)}$ em trabalhos onde maior número de fragmentos ${ }^{(21)}$ e de cortes seriados (70 por bloco) foi utilizado. Empregando esta mesma metodologia, ALCÁNTARA et al. ${ }^{(1)}$ encontraram granulomas em apenas $19,5 \%$, o que poderia ser explicado, segundo os autores, pela menor idade dos pacientes estudados e menor proporção de lesões endoscópicas, situações em que a incidência de granulomas é menor ${ }^{1}$, ${ }^{33)}$. Outros achados sugestivos de DC são linfangiectasias e folículos linfóides na profundidade da mucosa, inflamação transmural, abscesso de criptas, displasia epitelial e fibrose mucosa e submucosa combi$\operatorname{nadas}^{(39)}$.

Estudos recentes têm relacionado o achado de gastrite focal Helicobacter pylori negativa como sugestivo de envolvimento gastroduodenal pela DC, ocorrendo em $34 \%{ }^{(15)}$ a $76 \%{ }^{(26)}$ dos pacientes com DC distal. PARENTE et al. ${ }^{(27)}$, recentemente publicaram estudo comparando os achados endoscópicos e histopatológicos de 141 pacientes com DC conhecida, com 79 pacientes com colite ulcerativa e 141 controles normais encontrando prevalência de gastrite focal Helicobacter pylori negativa em 43\%, 12\% e 19\%, respectivamente, o que foi estatisticamente significativo. Os autores concluíram que este achado é freqüente em pacientes com DC e pode ajudar no diagnóstico diferencial das colites indeterminadas, embora não possa ser considerado como patognomônico da DC, enfatizando a importância da endoscopia digestiva alta com múltiplas biopsias na avaliação inicial destes pacientes.

A prevalência da infecção pelo Helicobacter pylori em pacientes com DC é descrita como menor que na população em geral, variando em torno de $30 \%{ }^{(5)}$. Sua presença dificulta a pesquisa de gastrite focal, uma vez que pode causá-la e, embora sua erradicação nestes casos não seja abordada pelos consensos, parece recomendável pois permitiria a análise das biopsias livre de seus efeitos patogênicos.

Os achados histopatológicos do duodeno são semelhantes àqueles descritos no estômago, sendo de interpretação mais fácil por não sofrerem interferências relacionadas à patogenicidade do Helicobacter pylori e da secreção cloridropéptica ${ }^{(40)}$.

O diagnóstico diferencial da DC gastroduodenal se faz, inicialmente, com doença péptica, devendo ser considerado também o carcinoma gástrico e de duodeno, gastroenterite eosinofílica, linfoma, pancreatite aguda, infecções granulomatosas (sífilis, tuberculose, histoplasmose), reações granulomatosas a corpos estranhos (berílio, silicato, talco), gastrite granulomatosa primária, sarcoidose, ingestão de soda cáustica, uso de reserpina, doença de Whipple e vasculite ${ }^{(3,7,8,18,38)}$.

A complicação mais freqüente é a obstrução, seguida de sangramento e formação de fístulas ${ }^{(24,31)}$, esta última ocorrendo geralmente a partir do segmento ileocólico doente, sendo rara a ocorrência de fístula primária a partir do segmento gastroduodenal ${ }^{(12)}$. Pancreatite aguda pode ocorrer, provavelmente secundária a refluxo através de um esfíncter de Oddi doente ou por fístulas ${ }^{(20)}$. O desenvolvimento de adenocarcinoma, embora controverso, pode ocorrer sendo descrito como raro ${ }^{(28)}$. 
O tratamento se baseia no uso de corticosteróides ${ }^{(16)}$, associados à drogas anti-secretoras potentes como os inibidores da bomba de prótons $^{(4)}$ que determinam acentuada melhora sintomática. Os salicilatos têm papel limitado nestes pacientes por apresentarem liberação mais distal e em relação aos imunossupressores e antibióticos, não existem dados suficientes que comprovem sua eficácia nesta forma da DC. SANDBORN ${ }^{(32)}$, utilizando FK 506 em três pacientes com DC proximal ou fistulizante, concluiu que a droga é clinicamente benéfica e útil como ponte para utilização de outros imunossupressores.

O tratamento cirúrgico é indicado nos pacientes com sangramento maciço, estenose progressiva, fistulização extensa e suspeita de câncer, ocorrendo em 10 a 40\% dos casos. O procedimento de escolha nos casos de obstrução é a gastrojejunostomia posterior uma vez que piloroplastia ou ressecção da área acometida pode levar à formação de fístulas. A vagotomia pode ser realizada no mesmo procedimento para reduzir o risco de úlcera marginal, que ocorre em 25 a 40\% dos pacientes, embora sua realização seja controversa pela possibilidade de diarréia no pós-operatório e da não proteção contra a mesma ${ }^{(11)}$.

Uma opção ao tratamento cirúrgico das estenoses é a dilatação endoscópica, eficaz nas estenoses curtas ${ }^{(17)}$, porém com recidivas freqüentes, o que é facilmente resolvido com dilatações repetidas, evitando-se a necessidade de novas intervenções cirúrgicas ${ }^{(22)}$.

Abrahão Jr LJ, Abrahão LJ, Vargas C, Chagas V, Fogaça H. Gastroduodenal Crohn's disease - report of four cases and review of the literature. Arq Gastroenterol 2001;38(1):57-62.

ABSTRACT - Background - Crohn's disease can affect all the gastrointestinal tract, but gastroduodenal involvement is rarely seen (0,5 to 13\%). Objectives - Report clinical, radiological and endoscopic findings and treatment of four patients with gastroduodenal Crohn's disease and review the literature. Patients and methods - Four patients (one male of 24 years old and three females of 37, 66 and 74 years old) with epigastric pain, weight loss and low grade fever were referred to the University Hospitals of Federal University of Rio de Janeiro and Fluminense Federal University. Two had also mild intermitent diarrhea and arthritis/arthralgia and the third developed pyloric obstruction and received surgical treatment. Anemia was observed in only one (the young female). Barium x-ray studies showed aphtous ulcers in stomach and duodenum with distal ileum lesions and deformity in both. Upper gastrointestinal endoscopy revealed aphtous ulcers in stomach and geographic duodenal ulcers. Polipoid lesions and serpiginous ulcers within gastric antrum were observed in the young female. Colonoscopy was performed in two patients and disclosed an ulcerated ileitis in one and ulcerated pancolitis in other. Histopathology findings of biopsy specimens were inconclusive (granulomas were not found) and other causes of granulomatous disease were ruled out. Corticosteroids and proton pump inhibitors were started and two patients had their disease controlled. The other patient developed pyloric obstruction and had to be operated. Conclusions - Gastroduodenal Crohn's disease has distinct clinical, therapeutic and prognostic features. Advances in endoscopic methods and recognition of new histopathologic criterias for diagnosis have revealed an incidence higher than previously reported.

HEADINGS - Crohn disease. Stomach diseases. Duodenal diseases.

\section{REFERÊNCIAS BIBLIOGRÁFICAS}

1. Alcántara M, Rodriguez R, Potenciano JL, Carrobles JL, Muñoz C, Gomez R. Endoscopic and bioptic findings in the upper gastrointestinal tract in patients with Crohn's disease. Endoscopy 1993;25:282-6.

2. Asquith P, Thompson RA, Cooke WT. Oral manifestations of Crohn's disease. Gut 1975;16:1249-54.

3. Bagby RJ, Rogers JV Jr, Hobbs C. Crohn's disease of the esophagus, stomach and duodenum: a review with emphasis on the radiographic findings. South Med J 1972;65:515-23.

4. Bianchi-Porro G, Ardizzone S, Petrillo M, Desideri S. Omeprazole for peptic ulcer in Crohn's disease. Am J Gastroenterol 1991;86:245-6.
5. D'Incà R, Sturniolo G, Cassaro M, di Pace C, Longo G, Callegari I, Rugge M. Prevalence of upper gastrointestinal lesions and Helicobacter pylori infection in Crohn's disease. Dig Dis Sci 1998;43:988-92.

6. Dancygier H, Frick B. Crohn's disease of the upper gastrointestinal tract. Endoscopy 1992;24:555-8.

7. Danzi JT, Farmer RG, Sullivan BH, Rankin GB. Endoscopic features of gastroduodenal Crohn's disease. Gastroenterology 1976;70:9-13.

8. Ectors NL, Dixon MF, Geboes KJ, Rutgeerts PJ, Desmet VJ, Vantrappen GR. Granulomatous gastritis: a morphological and diagnostic approach. Histopathology 1993;23:55-61.

9. Fielding JF, Cooke WT. Peptic ulceration in Crohn's disease (regional enteritis). Gut 1970;11:998-1000. 
10. Fielding JF, Toye KM, Beton DC, Cooke WT. Crohn's disease of the stomach and duodenum. Gut 1970;11:1001-6.

11. Glotzer DJ. The surgical management of idiopatic inflamatory bowel disease. In: Kirsner JB, Shorter RG, editors. Inflammatrory bowel disease. 3.ed. Philadelphia: Lea \& Febiger; 1988. p.590-1.

12. Greenstein AJ, Present DH, Sachar DB, Slater G, Heimann T, Lachman P, Aufses AH Jr. Gastric fistulas in Crohn's disease. Report of cases. Dis Colon Rectum 1989;32:888-92.

13. Gschwantler M, Kogelbauer G, Klose W, Bibus B, Tscholakoff D, Weiss W. The pancreas as a site of granulomatous inflammation in Crohn's disease. Gastroenterology 1995;108:1246-9.

14. Haggitt RC, Meissner WA. Crohn's disease of the upper gastrointestinal tract. Am J Clin Pathol 1973;59:613-22.

15. Halme L, Kärkkäinen P, Rautelin H, Kosunen TU, Sipponen P. High frequency of Helicobacter negative gastritis in patients with Crohn's disease. Gut 1996;38:379-83.

16. Haray AM, Rogers AI. Gastroduodenal Crohn's disease: differential diagnosis and treatment. Postgrad Med 1983;74:129-34.

17. Kelly SM, Hunter JO. Endoscopic balloon dilatation of duodenal strictures in Crohn's disease. Postgrad Med J 1995;71:623-4.

18. Khan MH, Lam R, Tamoney HJ. Isolated granulomatous gastritis: report of a case simulating gastric carcinoma. Am J Gastroenterol 1979;71:90-4.

19. Korelitz BI, Waye JD, Kreuning J, Sommers SC, Fein HD, Beeber J, Gelberg BJ. Crohn's disease in endoscopic biopsies of the gastric antrum and duodenum. Am J Gastroenterol 1981;76:103-9.

20. Legge DA, Hoffman HN 2d, Carlson HC. Pancreatitis as a complication of regional enteritis of the duodenum. Gastroenterology 1971;61:834-7.

21. Levine MS. Crohn's disease of the upper gastrointestinal tract. Radiol Clin North Am 1987;25:79-91.

22. Matsui T, Hatakeyama S, Ikeda K, Yao T, Takenaka K, Sakurai T. Long-term outcome of endoscopic balloon dilation in obstructive gastroduodenal Crohn's disease. Endoscopy 1997;29:640-5.

23. Nelson SW. Some interesting and unusual manifestations of Crohn's disease ("regional enteritis") of the stomach, duodenum and small intestine. Am J Roentgenol Radium Ther Nucl Med 1969;107:86-101.

24. Nugent FW, Richmond M, Park SK. Crohn's disease of the duodenum. Gut 1977;18:115-20.

25. Nugent FW, Roy MA. Duodenal Crohn's disease: an analysis of 89 cases. Am J Gastroenterol 1989;84:249-54.

26. Oberhuber G, Püspök A, Oesterreicher C, Novacek G; Zauner C, Burghuber M, Vogelsang H, Potzi R, Stolte M, Wrba F. Focally enhanced gastritis: a frequent type of gastritis in patients with Crohn's disease. Gastroenterology 1997; 112:698-706.

27. Parente F, Cucino C, Bollani S, Imbesi V, Maconi G, Bonetto S, Vago L, Porro GB. Focal gastric inflammatory infiltrates in inflammatory bowel diseases: prevalence, immunohistochemical characteristics, and diagnostic role. Am J Gastroenterol 2000;95:705-11.

28. Patel M, Banerjee B, Block JG, Marshall JB. Gastric Crohn's disease complicated by adenocarcinoma of the stomach: case report and review of the literature. Am J Gastroenterol 1997;92:1368-71.

29. Pinto MF. Doença inflamatória intestinal: a casuística do Hospital Universitário [dissertação]. Rio de Janeiro: Universidade Federal do Rio de Janeiro; 1998.

30. Post AB, Van Stolk R, Broughan TA, Tuthill RJ. Crohn's disease of the gallbladder. J Clin Gastroenterol 1993;16:139-42.

31. Rutgeerts P, Onette E, Vantrappen G, Geboes K, Broeckaert L, Talloen L. Crohn's disease of the stomach and duodenum: a clinical study with emphasis on the value of the endoscopy and endoscopy biopsies. Endoscopy 1980;12:288-94

32. Sandborn WJ. Preliminary report on the use of oral tacrolimus (FK506) in the treatment of complicated proximal small bowel and fistulizing Crohn's disease. Am J Gastroenterol 1997;92:876-9.

33. Schmitz Moormann P, Malchow H, Pittner PM. Endoscopic and bioptic study of the upper gastrointestinal tract in Crohn's disease patients. Pathol Res Pract 1985; 179:377-87.

34. Scully C, Cochran KM, Russell RI, Ferguson MM, Ghouri MA, Lee FD, Mac Donald DG, McIntyre PB. Crohn's disease of the mouth: an indicator of intestinal involvement. Gut 1982;23:198-201.

35. Stevenson GW, Laufer I. Duodenum. In: Laufer I, editor. Double contrast gastrointestinal radiology with endoscopic correlation. Philadelphia: Saunders; 1979. p.356-60

36. Tanaka M, Kimura K, Sakai H, Yoshida Y, Saito K. Long-term follow-up for minute gastroduodenal lesions in Crohn's disease. Gastrointest Endosc 1986;32(3):206-9.

37. Tishler JMA, Helman CA. Crohn's disease of the esophagus. J Can Assoc Radiol 1984;35:28-30.

38. Wagtmans MJ, Verspaget HW, Lamers CBHW, Van Hogezand RA. Clinical aspects of Crohn's disease of the upper gastrointestinal tract: a comparison with distal Crohn's disease. Am J Gastroenterol 1997;92:1467-71.

39. Wise L, Kyriakos M, McCown A, Ballinger WF. Crohn's disease of the duodenum: a report and analysis of eleven new cases. Am J Surg 1971;121:184-94.

40. Yardley J, Hendrix TR. Gastroduodenal Crohn's disease: the focus is on focality [editorial]. Gastroenterology 1997;112:1031-3.

41. Yokota K, Saito Y, Einami K, Ayabe T, Shibata Y, Tanabe H, Watari J, Ohtsubo C, Miyokawa N, Kohgo Y. A bamboo joint-like appearance of the gastric body and cardia: possible association with Crohn's disease. Gastrointest Endosc 1997;46:268-72. 